https://doi.org/10.21670/ref.1804004

Artículos

\title{
Paradiplomacia y desarrollo económico en la región transfronteriza de Reynosa-McAllen
}

\section{Paradiplomacy and economic development in the Reynosa-McAllen cross-border region}

Karla María Nava Aguirre $\mathrm{a}^{*}$ (iD) https://orcid.org/0000-0001-7072-8943 Gustavo Córdova Bojórquez b (ib) https://orcid.org/0000-0001-9085-1375

Recibido el 14 de julio de 2017. Aceptado el 8 de noviembre de 2017.

*Autor para correspondencia: Karla María Nava Aguirre, correo electrónico: karla.nava@udem. edu
Esta obra está protegida bajo una Licencia Creative Commons Atribución-NoComercial 4.0 Internacional.

\footnotetext{
${ }^{a}$ Universidad de Monterrey, Departamento de Economía de la División de Negocios, México, correo electrónico: karla.nava@udem.edu

${ }^{\mathrm{b}}$ El Colegio de la Frontera Norte, Departamento de Estudios Urbanos y del Medio Ambiente, Ciudad Juárez, Chihuahua, México, correo electrónico: gcordova@colef.mx
}

\section{Resumen}

La paradiplomacia es un nuevo fenómeno de participación internacional de gobiernos locales y otras entidades no-estatales. La investigación tiene como objetivo analizar la construcción paradiplomática y observar el desarrollo económico en la región transfronteriza Reynosa-McAllen a partir del Tratado de Libre Comercio de América del Norte (TlaAn). Se utilizó la investigación cualitativa con un enfoque descriptivo. Los instrumentos de recolección de información fueron la revisión bibliográfica y la entrevista. Los hallazgos demuestran que las actividades de vinculación entre Reynosa y McAllen, obedecen al trabajo en equipo que se realiza principalmente entre el Gobierno Municipal de Reynosa, Gobierno del Estado de Tamaulipas y la organización McAllen Economic Development Corporation (MEDC), que han incrementado la inversión y empleo generando un desarrollo económico en esta región transfronteriza.

Palabras clave: paradiplomacia, gobierno local, región transfronteriza, desarrollo económico.

\section{Abstract}

Paradiplomacy is a new international participation phenomenon involving local governments and other non-State entities. This research aims at analyzing paradiplomacy and observing the economic development in the Reynosa-McAllen cross-border region after the North American Free Trade Agreement (NAFTA). A qualitative research method with a descriptive approach was used. The data collection instruments were a literature review and interviews. The findings indicate that outreach activities between

CÓMO CITAR: Nava, K. y Córdova, G. (2018). Paradiplomacia y desarrollo económico en la región transfronteriza de Reynosa-McAIlen [Paradiplomacy and economic development in the Reynosa-McAllen cross-border region]. Estudios Fronterizos, 19 , e004. doi:10.21670/ref.1804004 
Reynosa and McAllen respond mainly to teamwork between the Municipal Government of Reynosa, the Tamaulipas State Government and the McAllen Economic Development Corporation (MEDC), which have increased investment and employment, thereby generating economic development in this cross-border region.

Keywords: paradiplomacy, local government, cross-border region, economic development.

\section{Introducción}

El mundo global que nos caracteriza hoy en día está cada vez más interconectado. "La multiplicación e intensificación de las transacciones económicas, políticas y sociales entre los Estados se le denomina Globalización" (Garza y Chacón, 2002, p. 3). Una de las modalidades de la globalización es, sin lugar a dudas, la internacionalización que a través de la acción exterior de los gobiernos se desarrollan estrategias de cooperación internacional en beneficio de los países y sus poblaciones. La falta de congruencia entre las políticas propuestas por el gobierno central y las necesidades de las entidades federativas, municipios, ciudades y condados, origina que estos se vinculen incluso con actores de otros países en la búsqueda de soluciones a sus problemáticas locales con o sin el respaldo de los gobiernos centrales. Solo en casos esporádicos el Estado ha considerado la vinculación internacional de los gobiernos no centrales con la política exterior (Cornago, 2010; Morales y Reyes, 2016).

A este fenómeno se le ha llamado Paradiplomacia, la cual, determina su proyección internacional de esos gobiernos subnacionales y de otros actores no estatales (Zeraoui, 2011; Rhi-Sausi y Oddone, 2013). Hoy en día, la paradiplomacia se puede describir como la participación de actores tradicionales como los gobiernos no centrales incluyendo los fronterizos y costeros, y de otros actores sociales públicos y privados que como agentes de presión impulsan la vinculación de sus actividades con el exterior creando redes de cooperación y contactos. Sin embargo, es importante resaltar que a pesar de los avances de la actividad paradiplomática, el papel del Estado sigue ejerciendo cierta influencia en las decisiones de algunos gobiernos locales o no centrales (Morales y Reyes, 2016).

Son varios los factores que se consideran claves en el desarrollo de la paradiplomacia. Aldecoa y Keating (1999) establecían tres aspectos que generan la actividad paradiplomática: los económicos, los políticos y los culturales. Entre estos factores se encuentra la globalización, que establece nuevas formas de interacción y vinculación entre los Estados y hacia el interior de los mismos (Garza y Chacón, 2002); la interdependencia económica, como consecuencia de los flujos crecientes de comercio entre países con redes globales de producción en sectores como el agrícola, manufacturas, turismo y servicios (Barajas, 2013); la competencia por los mercados internacionales de ciudades o regiones con características muy específicas o para atraer inversión; la apertura democrática de sistemas políticos y debilitamiento del Estado central (Zeraoui, 2011); la migración, generando grupos de presión y el interés de gobiernos locales por proteger sus derechos (Dávila, Schiavon y Velázquez, 2008), y el escenario fronterizo contemporáneo con distintas dinámicas y nuevos actores que compiten frente a las estrategias de colaboración en la frontera (Amilhat, 2016) entre otros. 
De esta forma, la paradiplomacia es una estrategia para poder negociar con actores internacionales en el marco de las relaciones entre dos o más países y está enfocada en obtener beneficios para los propios gobiernos locales o subnacionales. Según Cornago:

[...] La implicación de los Gobiernos No Centrales en las relaciones internacionales, a través del establecimiento de contactos formales e informales, permanentes o ad hoc con entidades extranjeras, públicas o privadas, con el propósito de promover diversos asuntos de carácter socioeconómicos, políticos o culturales, así como cualquier otra dimensión externa de sus propias competencias constitucionales (citado en Zeraoui, 2011, p. 70).

Por el lado del desarrollo económico, la región transfronteriza ${ }^{1}$ de ReynosaMcAllen presenta un despunte en algunos sectores económicos como el de manufactura, el de comercio y el de transporte a partir del TLCAN en 1994. A partir del acuerdo comercial, se conjuntan tres tipos de ejes en torno a los cuales gira la vinculación internacional, a saber: la política exterior federal, la transfronteriza y las que desarrollen con otras instancias o áreas más allá de ámbito estrictamente de frontera (Martínez, 2011). De acuerdo con Rhi-Sausi y Oddone (2013), la integración regional y actividades internacionales involucran la participación de nuevos actores como son los empresarios, universidades, organizaciones civiles (actores privados) y el fortalecimiento de la acción internacional de los gobiernos subnacionales en busca de complementariedades en dichos procesos de integración. Por lo tanto, las regiones transfronterizas son prioritarias en los procesos de cooperación e integración de dos países de acuerdo con su relevancia económica, política, social, cultural o ambiental. De acuerdo con Amilhat (2016) las fronteras no solo se concentran en los límites territoriales, sino en aquellas "fronteras móviles" que implican distinguir, pero sobretodo articular los flujos de personas, bienes y capitales entre otros. Se trata de ver a las fronteras no solo como instituciones sino como espacios sociales con multitud de actores.

En el área de influencia de esta región se toma en cuenta lo que se ha venido construyendo por ambos países en las últimas décadas, a saber: 17 cruces internacionales que favorecen el movimiento de mercancías y de personas, donde el flujo comercial se da de la siguiente manera: $52 \%$ por ferrocarril y $39 \%$ por carretera. Tamaulipas es la puerta a un comercio de 350000000 de consumidores y el cruce de más de 33\% del comercio internacional de México en el mundo. El estado de Tamaulipas contribuye a este comercio internacional gracias a la instalación de más de 350 empresas maquiladoras de exportación con más de 205000 empleos directos. Tamaulipas es el quinto exportador en México con 27423025 billones de dólares (Secretaría de Desarrollo Económico, 2017). El estado de Texas constituye una de las economías más grandes del mundo ${ }^{2}$ por lo que importa gran parte de la producción que viene del norte de México.

\footnotetext{
${ }^{1}$ De acuerdo con Perkmann (citado por Oliveras, Durà y Perkman, 2010, p. 24) una región transfronteriza se define como la "unidad territorial delimitada compuesta por las autoridades territoriales participantes en una acción de cooperación transfronteriza”.

${ }^{2}$ Texas cuenta con una población total de 26956958 habitantes y un crecimiento de $7.2 \%$ en donde su población se considera joven. El producto interno bruto del estado es de 1532623 millones de dólares (2013), solo después de California (Alonso, 2015).
} 
A partir de este contexto, sostenemos que el desarrollo económico de la región transfronteriza Reynosa-McAllen se debe en gran medida a la paradiplomacia y con base en ello surge la siguiente interrogante: ¿De qué manera se ha construido la paradiplomacia y cómo esta contribuye al desarrollo económico en la región transfronteriza Reynosa-McAllen? Se plantean dos objetivos para el presente estudio; en primer lugar, analizar la construcción de la paradiplomacia y en segundo lugar, observar el desarrollo económico de esta región transfronteriza a partir de la práctica de esta, en el contexto del TLCAN.

Las ciudades seleccionadas para la presente investigación son relevantes ya que Reynosa es la ciudad más poblada de la región fronteriza de Tamaulipas, México y es a su vez la que presenta el mayor dinamismo económico dado el número de industrias maquiladoras y los empleos generados en este sector. Por el otro lado, la ciudad de McAllen junto con Harlingen, son los centros urbanos que presentan el mayor crecimiento regional en Texas, Estados Unidos (McAllen Chamber of Commerce, 2016).

La investigación que acompaña este trabajo es de tipo cualitativo con alcance descriptivo-exploratorio. Las técnicas de recolección de información fueron en primer lugar, la revisión bibliográfica de fuentes secundarias de información, principalmente documentos impresos, bases de datos, artículos, libros, proyectos y estudios vinculados al tema de paradiplomacia, vinculación internacional y cooperación transfronteriza y, en segundo lugar, la entrevista semiestructurada realizada a actores clave de ambas ciudades. ${ }^{3}$ La meta de esta primera aproximación, ya que la investigación también abarca otros pares de ciudades fronterizas, fue obtener información relacionada con las actividades de vinculación tanto de Reynosa como McAllen y destacar la participación de la región fronteriza en los procesos de cooperación internacional.

De esta manera, el trabajo se divide en cuatro apartados. El primero, describe brevemente la región transfronteriza Reynosa-McAllen. El segundo, analiza la acción exterior de los gobiernos locales y su actividad paradiplomática. El tercero, describe el impacto en el desarrollo económico y, en el cuarto, se vierten las conclusiones.

\section{Región transfronteriza Reynosa-McAllen}

Tamaulipas es uno de los seis estados fronterizos de México. Se localiza en el centro del flujo comercial de Norteamérica, con acceso al mercado más dinámico del mundo (Figura 1). Comparte $370 \mathrm{~km}$ de frontera con Estados Unidos (Texas). Tiene una superficie de $80174 \mathrm{~km}^{2}$ y es el sexto estado más grande del país. Cuenta con una población de 3.4 millones de habitantes, su población económicamente activa es de 1.3 millones, es decir, 38\% de la población total (Secretaría de Desarrollo

\footnotetext{
${ }^{3}$ El primer actor entrevistado fue el Sr. Ralph García, Vicepresidente de Negocios en México del McAllen Economic, Development Corporation (MEDC). El Sr. García cuenta con más de 15 años de experiencia en el desarrollo económico de la zona y es el vínculo principal con la oficina de Desarrollo Económico del Municipio de Reynosa. El segundo actor entrevistado fue el Lic. Vicente Valdez Gutiérrez, Secretario de Desarrollo Económico del Gobierno Municipal de Reynosa y el tercer actor entrevistado es el Contador Público Ángel Ortiz Salazar, encargado del despacho de la Secretaría de Desarrollo Económico y Turismo (SEDET) del Gobierno del Estado de Tamaulipas en Ciudad Victoria.
} 
Económico, 2017). Esta entidad federativa es una de las nueve economías más importantes del país y aporta $3 \%$ del producto interno bruto. Según el reporte de la Comisión Nacional de Inversiones Extranjeras (2016) para finales de diciembre de 2016, Tamaulipas ocupaba el $7^{\circ}$ lugar destino en México para la inversión extranjera.

Figura 1: Ubicación geográfica de Tamaulipas

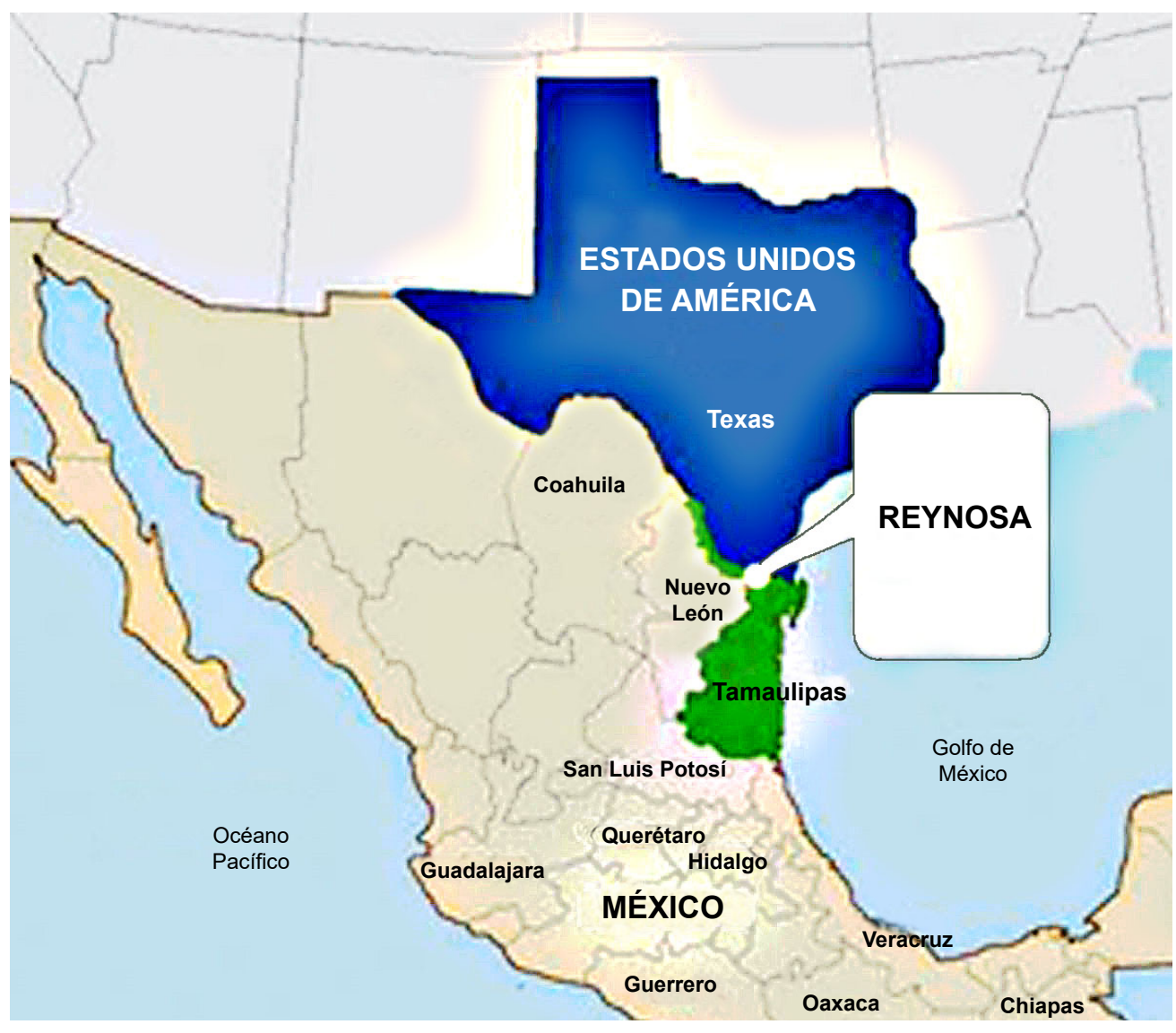

Fuente: Consejo Binacional para el Fomento Económico de Reynosa (Cobifer), 2013.

El estado está dividido en seis regiones, a saber: Región Frontera, Valle de San Fernando, Centro, Altiplano, Mante y Sur (Figura 2). Reynosa es uno de los diez municipios fronterizos junto con Matamoros, Valle Hermoso, Río Bravo, Gustavo Díaz Ordaz, Camargo, Miguel Alemán, Mier, Guerrero y Nuevo Laredo que conforman la Región Frontera. De estos municipios, las ciudades con una población mayor a los 350000 habitantes en el año 2010 según datos del Instituto Nacional de Estadística, Geografía e Informática (Inegi, 2016b) son Reynosa con 608 891, ${ }^{4}$ Matamoros con 489193 y Nuevo Laredo con 384033 habitantes.

\footnotetext{
${ }^{4}$ Según el conteo intercensal de 2015, Reynosa alcanzó los 646202 habitantes (Inegi, 2015).
} 
Figura 2: Regiones de Tamaulipas

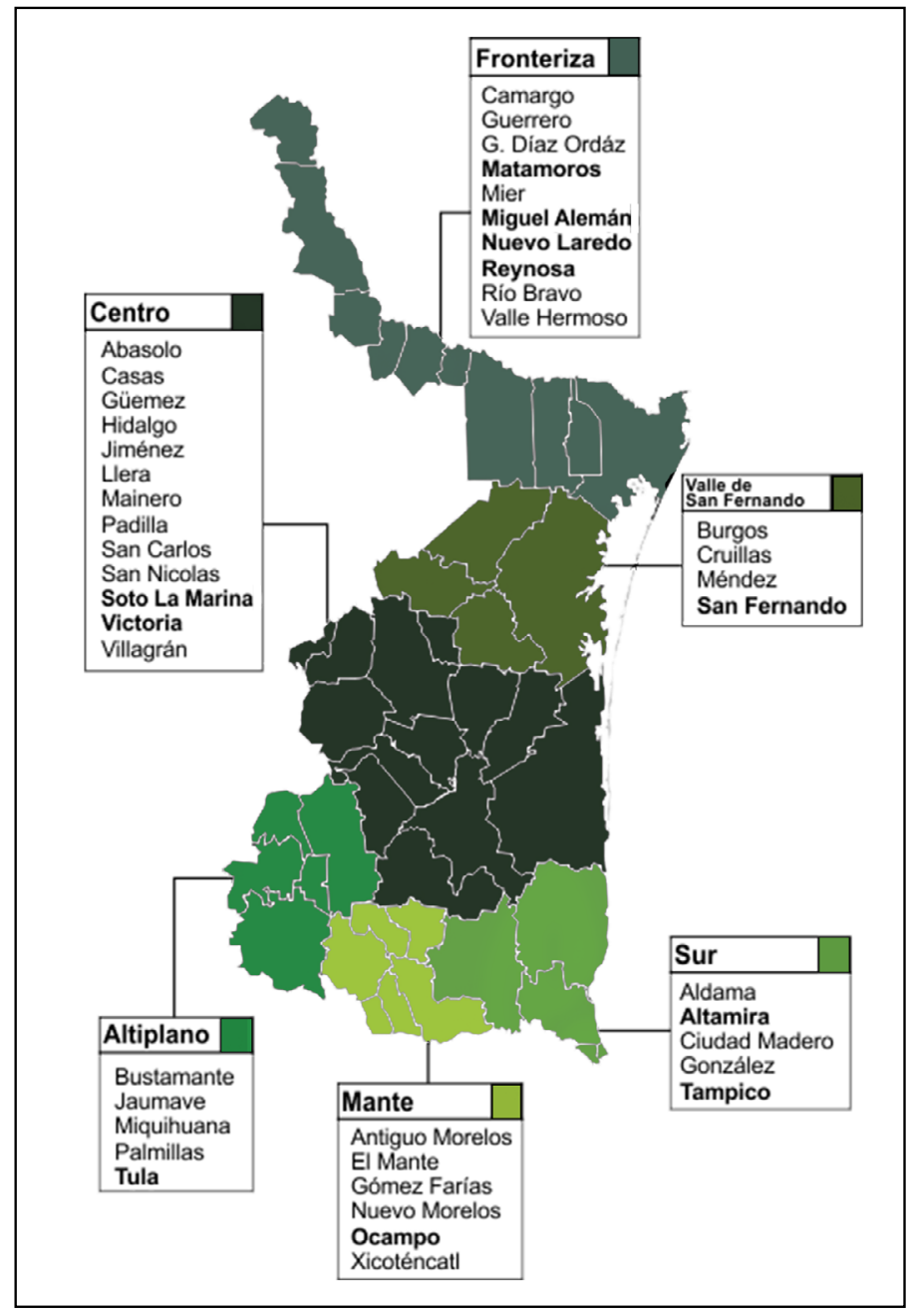

Fuente: Gobierno del Estado de Tamaulipas (s.f.).

Gracias al TLCAN, la vocación de la región fronteriza es la logística de comercio exterior, la industria manufacturera, en especial las relacionadas con lo eléctricoelectrónico y la de autopartes. Asimismo, hay otras actividades que acompañan a esta actividad principal como el turismo cinegético, el de negocios y el de salud (Plan Estatal de Desarrollo Tamaulipas (2011-2016), 2013). El último reporte oficial indica que la industria manufacturera en el estado tiene instaladas 350 empresas y genera 205514 empleos directos; donde Reynosa, tiene instaladas a 147 empresas y genera 97118 empleos directos (Inegi, 2016a). Esta ciudad cuenta con una infraestructura importante para desarrollar la industria manufacturera, como es el hecho de contar con 14 parques industriales y tres puentes internacionales que facilitan el cruce tanto de mercancías como de personas diariamente (Figura 3). Los puentes en cuestión son el Cruce Internacional Anzaldúas, el Puente Internacional McAllen-Hidalgo-Reynosa y el Puente Reynosa-Pharr, este último facilita principalmente el cruce de carga. 
Figura 3: Parques industriales y puentes internacionales de Reynosa

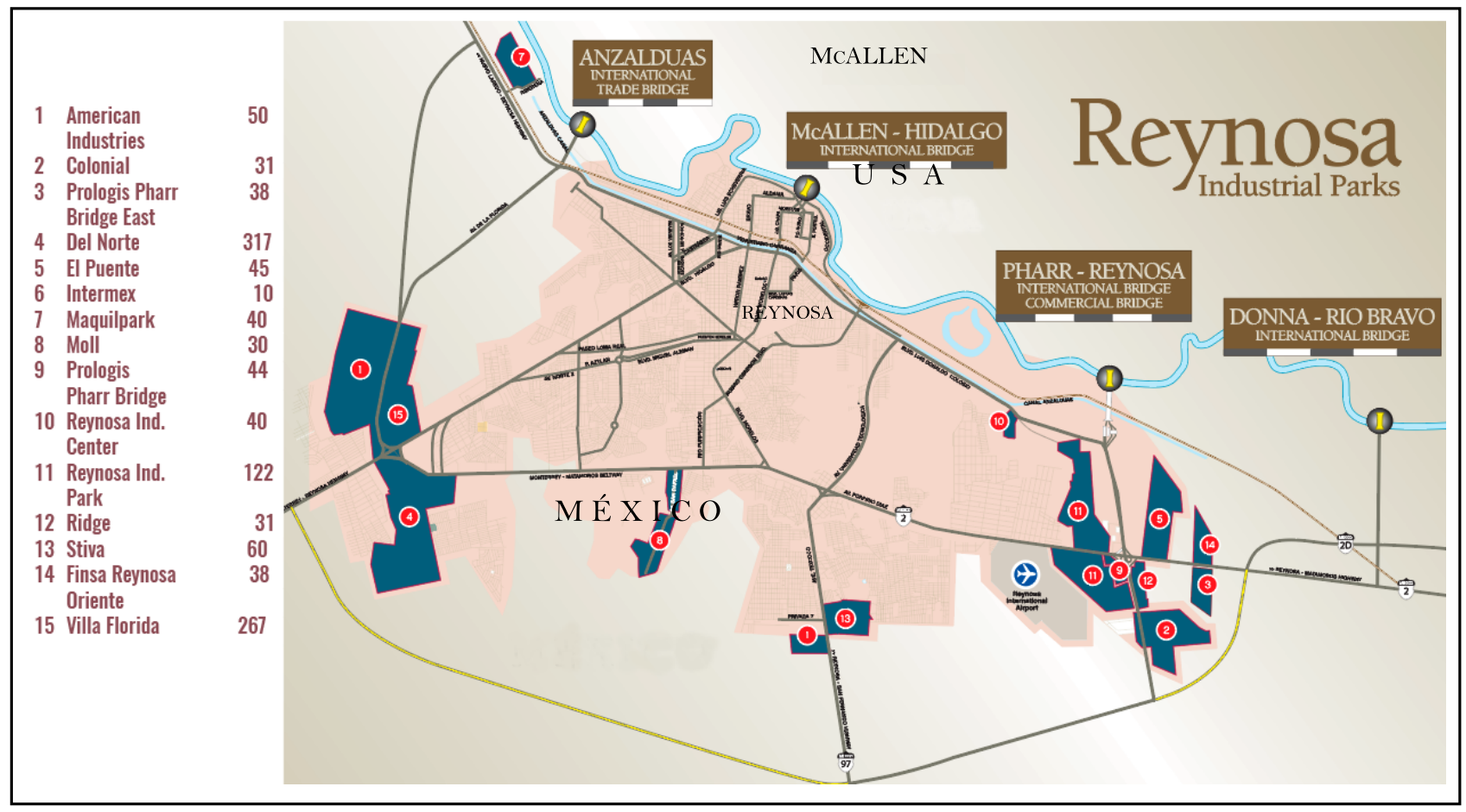

Fuente: McAllen Economic Development Corporation (s.f.).

Por la parte de Texas, la ciudad de McAllen pasa de ser una ciudad con una economía basada en la agricultura y ganadería a una ciudad con vocación al comercio exterior, salud, comercio y turismo. La cercanía y lazos con México propiciaron que la ciudad creciera y rápidamente se transformara como un puerto de entrada para los viajeros en ambos lados de la frontera. Durante los ochenta y noventa, la floreciente industria maquiladora en Reynosa provocó el desarrollo de la zona de libre comercio de McAllen así como el interés de potenciar la zona.

De acuerdo con el censo 2010, se registró una población de 774769 habitantes en la región de McAllen, Edinburg y Mission, es decir, un incremento de 66.8\% con respecto al 2001. La región Reynosa-McAllen es considerada uno de los centros urbanos más dinámicos en la frontera México-Estados Unidos. En este contexto, McAllen Economic Development Corporation (MEDC) ha jugado un rol importante en la promoción del desarrollo económico, no solo de McAllen, sino también de Reynosa. MEDC es una organización sin fines de lucro fundada hace más de 29 años por los señores Mike Allen (finado) y Keith Patridge (actual presidente), hombres de negocios con la visión de generar un crecimiento industrial, por ello, la meta del MEDC es promover el desarrollo económico de la zona a través de la atracción de nuevas industrias a McAllen y Reynosa con un enfoque totalmente industrial. El slogan de la organización "Dos naciones, una ciudad" pone en evidencia la visión de esta organización transfronteriza. 
Por su parte, en la ciudad de Reynosa, la oficina encargada de dichas acciones de promoción industrial, es la Secretaría de Desarrollo Económico del Gobierno Municipal de Reynosa. Para fortalecer lo anterior, el Gobierno del Estado de Tamaulipas a través de la Secretaría de Desarrollo Económico y Turismo (SEDET) es la dependencia encargada de promover el desarrollo económico de todo el estado y cada uno de sus 43 municipios.

Como se ha podido observar, las dos ciudades en cuestión se han venido fortaleciendo en el campo económico, en especial, a partir del TLCAN y por el trabajo de varios actores de la sociedad de ambos lados que han logrado avanzar, sin duda en el campo de la paradiplomacia.

\section{Paradiplomacia en la región Reynosa-McAllen}

Como ya se mencionó, la paradiplomacia se refiere a un nuevo fenómeno de participación internacional de los gobiernos locales y otras entidades no estatales. El TlCAN, fue un detonante de la actividad internacional en México, debido a la importancia que se le dio al tema económico por encima de otros aspectos (González, 2002). Se destaca que la región noreste de México cuenta con un desarrollo diferente al resto del país y una posición geográfica excepcional, al ser frontera con el país más poderoso del mundo en términos comerciales y políticos, por lo que las oportunidades de desarrollo se encuentran precisamente hacia al norte, ofreciendo productos y servicios (Soldatos, citado en Zeraoui, 2011). Oliveras (2014) utiliza el concepto de paradiplomacia para definir el conjunto de contactos, actividades, procesos e iniciativas exteriores entre los gobiernos locales y de dichos gobiernos con otros actores públicos y privados tal como lo describen también Aldecoa y Keating (2001), Duchacek (1986) y García (1996). En otras ideas Amilhat (2016) describe la paradiplomacia contemporánea a partir de la globalización competitiva de los territorios en donde la frontera participa. En la región existen en efecto, variadas actividades que forman parte de la paradiplomacia como son: la cooperación territorial, cooperación al desarrollo, acuerdos de hermanamiento de ciudades, las visitas de autoridades gubernamentales al exterior y la recepción de autoridades públicas y privadas extranjeras; campañas de promoción industrial, turística y comercial y de difusión cultural en eventos internacionales; participación en asociaciones y foros internacionales, todas ellas como estrategias de desarrollo local desde la arena internacional.

Cobra especial relevancia el hermanamiento de ciudades que incrementa el flujo de información y cooperación en actividades específicas relacionadas con el desarrollo de la región transfronteriza en un contexto global (Lecours, 2002; Zeraoui, 2011). Para lograr esto, los gobiernos locales disponen de recursos financieros, políticos, técnicos y tecnológicos para estar en contacto con actores de otros países (Gutiérrez, 2013), lo que suple de alguna manera, la falta de atención de los gobiernos centrales. Así, ante la falta de interés del gobierno central por la problemática que enfrentan los gobiernos locales, estos últimos optan por vincularse con otros gobiernos locales más cercanos, como sería la cooperación transfronteriza en aquellos municipios de la frontera norte mexicana. A modo de ejemplo, se puede mencionar la Asociación de Alcaldes de la Frontera Norte de México, 
A.C., organismo que inició sesiones en el año 2007 en la ciudad de Tijuana, Baja California y constituyéndose como asociación civil en el año 2010. De este esfuerzo se han generado acuerdos generales para la solución de problemáticas de la región en temas de seguridad, infraestructura, migración, relaciones internacionales, municipalismo, medio ambiente, desarrollo económico y salud (Córdova, 2013).

En el caso de la región, Oliveras (2014) es enfático en señalar que la participación es cada vez más activa de los gobiernos locales, principalmente los municipios de Nuevo Laredo, Matamoros y Reynosa y la relación con sus ciudades hermanas de Laredo, Brownsville y McAllen respectivamente. A pesar del ambiente en ocasiones tan adverso en dicha región, se mencionan una serie de vínculos entre las ciudades además de los acuerdos de hermanamiento aunque no exhaustivo. ${ }^{5}$

Otra vertiente que interesa resaltar para esta región transfronteriza es la legal, donde al menos para México, ha habido un viraje en la relación con los Estados Unidos. Ahora se reconoce que actores locales pueden avanzar y madurar la relación sin olvidar que pertenecen a un Estado nación que los puede apoyar y, en cierto momento, facilitar la interacción con actores sociales del otro lado. La Ley sobre Celebración de Tratados (1992) en el artículo $1^{\circ}$ establece que los acuerdos interinstitucionales podrán celebrarse entre una dependencia u organismo descentralizados de la administración pública federal, estatal o municipal y uno o varios órganos extranjeros $\mathrm{u}$ organizaciones internacionales.

Se define a un acuerdo interinstitucional como el nombre genérico para cualquier documento que se suscribe con un órgano gubernamental extranjero u organismo internacional, independientemente de su denominación (acuerdo de cooperación, convenio, protocolo, acuerdo de hermandad, etc.). Dicha Ley, convirtió a los gobiernos estatales y municipales en sujetos de derecho internacional. Además, se estableció que la Secretaría de Relaciones Exteriores (SRE) debía ser informada sobre cualquier acuerdo interinstitucional (Rodríguez, 2006). ${ }^{6}$ Este marco legal, viene a reconocer el trabajo que se hace en el nivel local, en especial, en regiones con alto dinamismo económico y social como lo es la frontera Reynosa-McAllen.

Ahora bien, en esta región una pluralidad de actores hacen promoción de iniciativas de cooperación transfronteriza con su contraparte norteamericana bajo el esquema de acuerdos interinstitucionales con el objetivo de promover el desarrollo regional y local tal como Ramos (2002) lo señala para los actores gubernamentales de la frontera México-Estados Unidos. Lo que se observa en esta dinámica, es la cooperación transfronteriza como una colaboración entre unidades subnacionales más allá de las fronteras nacionales de un país que permite la participación y actuación conjunta, en forma de red con los actores públicos y privados del territorio en ambos lados de la frontera (Oddone, 2014). Este autor sostiene que: "su objetivo

\footnotetext{
${ }^{5}$ Para Oliveras (2014) el estudio de la acción transfronteriza es un esfuerzo por analizar la participación de los gobiernos locales en actividades vinculadas con el exterior, un tema tratado históricamente a través de las políticas establecidas o formuladas desde los gobiernos centrales o del Estado nación. Se destaca que la acción exterior de los gobiernos locales es un tema poco estudiado, idea que se comparte con las aportaciones de Dávila et al. (2008), Kincaid (1999), Schiavon (2006) y Velázquez (2007).

${ }^{6}$ La Secretaría en su Reglamento Interior artículo $16^{\circ}$ establece que corresponde a la Dirección General de Coordinación Política promover el establecimiento de mecanismos de coordinación entre la Secretaría y las oficinas que manejan asuntos internacionales en otras áreas de la administración pública federal, de las entidades federativas y municipales (Rodríguez, 2006).
} 
global es que las relaciones entre poderes territoriales vecinos se desarrollen en la misma naturalidad que si la frontera no existiese" (Oddone, 2014, p. 132).

La actividad paradiplomática en la región la podemos describir también desde el punto de vista económico, político y social-cultural.

Desde el punto de vista económico, la actividad paradiplomática en la región se remonta a varias décadas. La globalización ocasionó que municipios fronterizos mexicanos utilizaran como estrategia la internacionalización de sus actividades para vincularse con el exterior. La firma del TLCAN fue uno de los factores que detonó la dinámica económica de esta región transfronteriza. El tratado, que fue negociado a finales de los años ochenta, se manifiesta en cada vez más empleos en la industria maquiladora de exportación, que junto con la paradiplomacia, es decir el intercambio entre actores locales, se han venido dando avances en materia económica de tal suerte que se ha entendido muy bien sus alcances como instrumento de desarrollo, para lo cual, los actores locales junto con el apoyo federal de ambos lados se han dado a la tarea de generar cada vez más infraestructura productiva como los tres puentes internacionales.

Tanto en Reynosa como en McAllen existen otros actores que participan directa e indirectamente en la vinculación internacional de la ciudad como son grupos de inversionistas, cámaras de comercio, asociaciones, universidades y consulados. Específicamente en Reynosa, la participación de los empresarios de distintos sectores lo constituyen el Consejo Binacional para el Fomento Económico de Reynosa (Cobifer), el Consejo Nacional para la Industria Maquiladora y Manufacturera de Exportación de Reynosa (Index) y la Cámara Nacional de la Industria de la Transformación de Reynosa (Canacintra).

Es importante señalar que las actividades paradiplomáticas se llevan a cabo principalmente a través del trabajo en conjunto del Municipio de Reynosa, el Gobierno de Tamaulipas y el MEDC. Para la Ciudad de McAllen, ha quedado claro que la manera de crecer como región es a través de la cooperación. La forma en que funciona la actividad paradiplomática es por medio de equipos de trabajo que se coordinan para compartir información, establecer puentes con sectores sociales de ambos lados, realizar tareas conjuntas, manteniendo la comunicación con otras regiones, dirimiendo asuntos contenciosos cuando se necesite, entre otras actividades.

De acuerdo con la experiencia del encargado del despacho de la SEDET, esta dinámica no es nueva, son ejercicios que se vienen desarrollando desde hace más de 25 años y todo obedece a los procesos de apertura y de globalización, pero sobre todo, a las necesidades de cada ciudad. Lo anterior se confirma con los inicios del MEDC, organismo que desde sus orígenes se ha vinculado con Reynosa para compartir y apoyar a la ciudad con el objetivo principal de atraer inversión. De igual forma, el gobierno de Reynosa confirma que estas actividades, si bien no son nuevas, se han acelerado desde finales de los noventa.

Desde el punto de vista político, la ciudad de Reynosa generó los Acuerdos Interinstitucionales de Amplio Alcance (AHAA), entre los que destacan los acuerdos de hermanamiento de ciudades. Según datos del gobierno de Reynosa, solo existen dos, uno firmado con la ciudad de McAllen y otro con Pharr, Texas. En ambos acuerdos, el Consulado de México en McAllen ha sido la instancia responsable de llevar a cabo la formalización del mismo a través de su inscripción en el Registro de Acuerdos Interinstitucionales (RAI) en la Dirección de Coordinación Política de la Secretaría de Relaciones Exteriores. 
En relación al AHAA Reynosa-McAllen, el día 27 de abril del 2016 se reunieron en la ciudad de McAllen, el alcalde de dicha ciudad Jim Darling y el de Reynosa, José Elías Leal en donde se reafirmó la resolución que existía desde el año de 1992 confirmando la relación tan estrecha entre estas ciudades hermanas con la intención de mantener la amistad y cooperación entre Reynosa y McAllen. El cónsul de México en McAllen, Guillermo Ordorica y la cónsul de Estados Unidos en Matamoros, Angela Kerwin firmaron como testigos. En ese día y como ejemplo de la buena voluntad y disposición de las ciudades hermanas, se hizo entrega de uniformes para los bomberos de Reynosa. Posteriormente, se realizó un intercambio de regalos entre los alcaldes.Entre los invitados estuvieron los directivos del MEDc, empresarios de ambos lados de la frontera, representantes de Index, Canacintra, Cobifer y la oficina de Desarrollo Económico Municipal.

Sin embargo, como lo afirma Ángel Ortiz de la SEDET en la entrevista realizada (2016), los acuerdos de hermanamiento o interinstitucionales no son indispensables para que el Gobierno del Estado promueva y apoye la vinculación de los municipios con el exterior. Estos sirven para confirmar y fortalecer la disposición de ambas ciudades y la confianza.

Derivado de este acercamiento, se realizan conjuntamente actividades de promoción industrial, comercial, turística y cultural a través de foros, seminarios, congresos, recepción de autoridades públicas y privadas extranjeras, visitas de autoridades gubernamentales al exterior, asistencia a ferias y reuniones anuales nacionales e internacionales. En relación con la promoción industrial de la zona, además de los Estados Unidos, países como Brasil, Japón, Corea y recientemente Italia se han convertido en objetivos de atracción de inversión.

Entre los últimos eventos que destaca Reynosa se encuentra la reunión de cámaras de comercio de Reynosa y McAllen para fortalecer lazos de colaboración y la reunión de alcaldes. Por otra parte, el MEDC, destaca las reuniones con el Index, la Canacintra, los torneos de golf y pícnic con las maquiladoras de Reynosa, la asistencia a conferencias binacionales y múltiples eventos de promoción de la zona Reynosa-McAllen dentro y fuera del país con el acompañamiento del Gobierno Municipal de Reynosa, Gobierno del Estado e inversionistas privados. Los eventos son organizados o promovidos por los distintos tipos de actores mencionados anteriormente, sin embargo, todos participan y todos tienen el mismo objetivo: promover y ofrecer la Región Reynosa-McAllen como una sola ciudad.

Por otra parte, existe una serie de eventos que la SEDET promueve en todos los municipios. En ocasiones, la labor de promoción la realiza el Estado y una vez detectados los prospectos se hace un acercamiento con los municipios. A partir del año 2011, la SEDET ha participado en 57 eventos nacionales e internacionales y se han atendido a más de 350 inversionistas. Entre las últimas misiones comerciales en el extranjero de esta dependencia estatal destacan las visitas a Francia, Italia, Japón, Corea, Alemania y los Estados Unidos.

Sin lugar a dudas, el trabajo realizado por el MEDC es muy significativo para las actividades paradiplomáticas de Reynosa tratando de fortalecer los aspectos económicos y políticos. La relación del organismo norteamericano con el gobierno municipal y estatal mexicano es muy estrecho y les ha quedado claro que la ciudad de McAllen necesita y depende en gran medida de la ciudad hermana de Reynosa para su crecimiento y busca seguir manteniendo una relación de confianza y respeto mutuo. 
Desde el punto de vista sociocultural, se han realizado acercamientos con las instituciones de educación superior con el objetivo de atender las demandas del sector industrial y capacitar y preparar a los próximos profesionistas con las competencias necesarias para incorporarse al ámbito laboral relacionado con la industria manufacturera. Recientemente, se logró un convenio con el South Texas College (STC) en McAllen y el Instituto Internacional de Estudios Superiores (IIES) de Reynosa Campus Villa Florida para capacitar a los estudiantes con la tecnología e instrumentos de la universidad texana, especialmente en el área de robótica, con el objetivo de desarrollar habilidades específicas de la industria para atender las necesidades del sector industrial. Aunque existen otras universidades tanto en Reynosa como McAllen, este convenio es pionero. El programa del Institute of Advanced Manufacturing del SCT es una de las iniciativas para capacitar con las habilidades técnicas y el conocimiento necesario a los estudiantes para enfrentar la competitividad; así que desde el año 2007, se han capacitado más de 27000 personas para operar procesos de manufactura y automatización en más de 200 empresas.

De manera similar, la University of Texas Rio Grande Valley (UTRVG) es otra institución educativa que trabaja en equipo con el MEDC y en beneficio de la región fronteriza. Su programa anual de una semana llamado Hispanic Engineering, Science, and Technology (HESTEC) es reconocido a nivel nacional como un modelo para promover la ciencia y tecnología en ambos lados. Lo anterior favorece los procesos de capacitación de la mano de obra especializada que se requiere en la región.

\section{Desarrollo económico transfronterizo}

De acuerdo con Barajas (2015) existen cuatro niveles de intervención en las relaciones transfronterizas entre México y los Estados Unidos: nivel trilateral (incluyendo a Canadá), nivel bilateral, nivel transfronterizo (estados y municipios en ambos lados de la frontera) y nivel local y estatal. ${ }^{7}$ En estos dos últimos niveles la actividad paradiplomática ha sido consistente por lo que el impacto en la zona ha sido positivo toda vez que lo que se promueve no son las ciudades en sí, sino una región transfronteriza como tal. En este sentido, las actividades de vinculación entre Tamaulipas y Texas han generado potenciar la zona del Valle del Río Grande o como dicen su pobladores "Mágico Valle del Río Grande" (Figura 4), zona que comprende los condados de Starr, Hidalgo, Willacy y Cameron que incluyen las ciudades de McAllen (Hidalgo), Edinburg, Harlingen, Brownsville y la Isla del Padre (Sandoval, 2007) y los municipios de Nuevo Laredo, Reynosa y Matamoros.

\footnotetext{
${ }^{7} \mathrm{Al}$ analizar el desarrollo fronterizo por etapas se establecen cinco periodos para su estudio. De 1848-1994 como una etapa conciliatoria; de 1942-1993, como una fase de programas de cooperación y redes transfronterizas; el período 1848-1994 caracterizado por sus políticas de complementariedad de la frontera norte; la etapa de 1961-1983 de integración internacional económica y finalmente la etapa de 1984-2008 de integración regional con América del Norte donde la firma del TLCAN es uno de los ejemplos más claros de la evolución del desarrollo fronterizo.
} 
Figura 4: Condados y ciudades del Valle de Río Grande

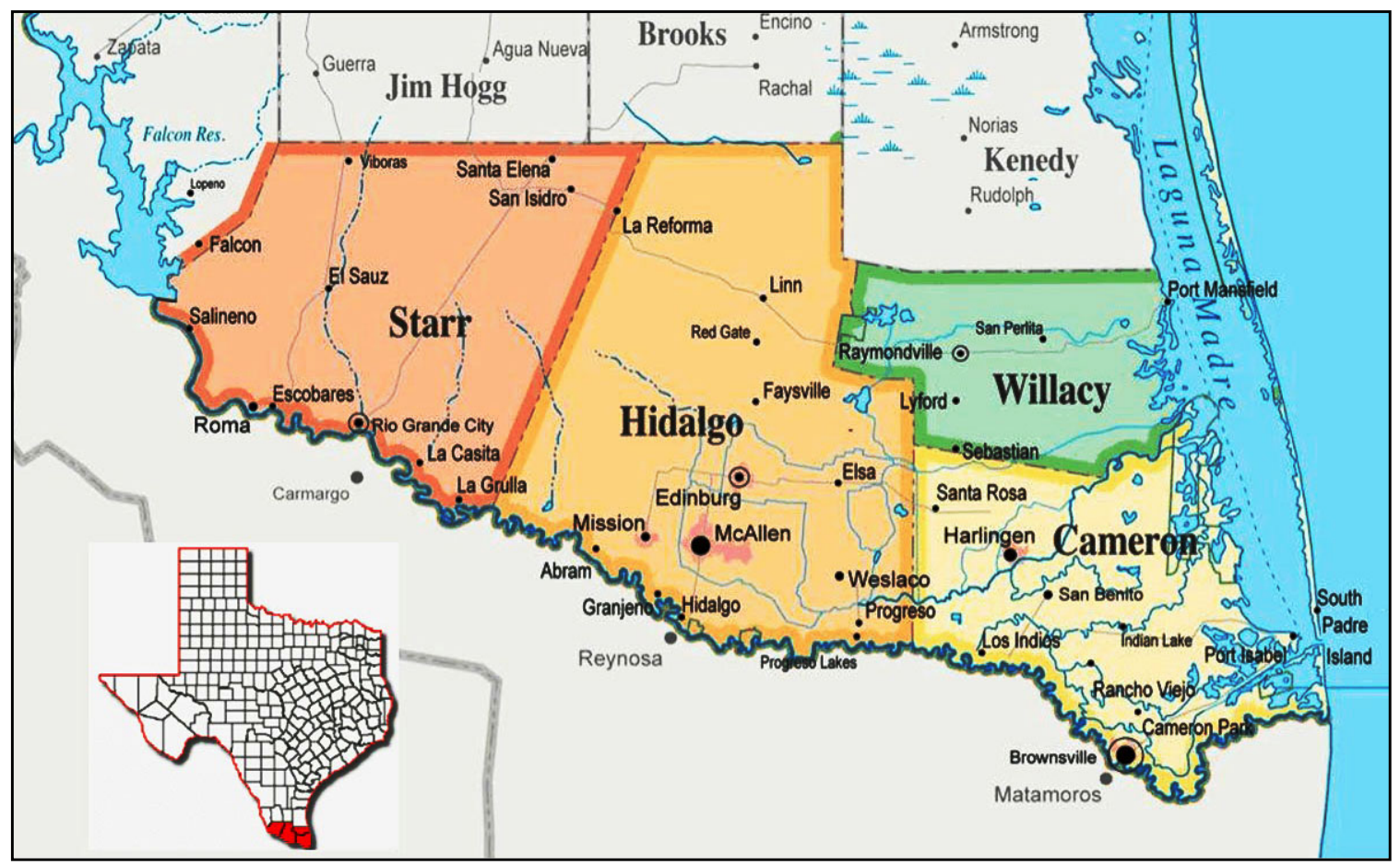

Fuente: Texas A\&M Agrilife (s.f.).

Lo que se ha podido constatar es que entre las autoridades gubernamentales tanto de municipio de Reynosa como del gobierno del estado, así como el MEDC en el lado norteamericano, existe un consenso en atribuir un desarrollo económico regional transfronterizo producto de la vinculación de las ciudades hermanas. Los aspectos para evaluar esa contribución al desarrollo de la zona son la inversión extranjera directa y los empleos generados por la misma. Existe un "efecto dominó" que ha permitido que esa dinámica de vinculación entre Reynosa y McAllen genere algo más allá de un crecimiento económico llegando a ser más visible en el lado norteamericano.

De acuerdo con el MEDc, Reynosa cuenta con mano de obra especializada, capacitada y con salarios competitivos. Solo en el año 2014, la tasa de ocupación en Reynosa respecto al porcentaje de su población económicamente activa fue de $96.4 \%$ esta cifra por encima de la media estatal de $60 \%$ y nacional de $59 \%$ (Primer Informe de Gobierno 2014 José Elías Leal. Presidente Reynosa Tamaulipas, 2014). En relación al sector educación, los grados promedios de escolaridad aprobados por la población de 15 años o más fue de 9.3, por encima de la media estatal y nacional. En relación con el ingreso y los derechos sociales, la medición de la pobreza en Reynosa se encuentra en $33.7 \%$ que aunque no es aliciente, se encuentra por debajo de la media estatal y nacional. Un dato importante que determina de alguna manera la calidad de vida en la ciudad de Reynosa, es el aumento en el número de verificaciones en las inspecciones ambientales en relación con las denuncias, las cuales, solo en 2014 fueron de 600, muy por encima de la media estatal de 103 (Primer Informe de Gobierno, 2014).

Según el MEDC, el impacto de las industrias que se instalan en Reynosa se traduce en empleo, desarrollo de proveedores, prestadores de servicios, personal especializado 
para incorporarlo al ámbito laboral tanto en el lado mexicano como norteamericano y una derrama económica por concepto de compras en tiendas departamentales, supermercados, restaurantes y el turismo en la ciudad de McAllen (García, 2016). Por lo anterior, sin el crecimiento económico y demográfico de la ciudad en Reynosa, no existiría un desarrollo en McAllen. Además, el impacto positivo también se refleja en las ciudades vecinas de Pharr, Mission y Edinburg. Por ejemplo, de cada 10 empleos generados en la industria maquiladora en Reynosa, se genera uno en McAllen (Ortiz, 2016).

Por lo anterior, la industria maquiladora es una parte importante de las economías en ambos lados de la frontera con más de un millón de trabajadores en esta industria. El flujo de inversión extranjera directa (IED) en Tamaulipas de 1999 a 2016 fue superior a los 1111 millones de dólares, esto representa cerca de $4.2 \%$ del total de la IED para México. De esta manera, el estado se ubica en el séptimo lugar nacional en captación y el cuarto de los estados de la frontera norte. La IED proviene de Estados Unidos, España y los Países Bajos en este orden (Comisión Nacional de Inversiones Extranjeras, 2016). En el primer semestre del 2017, Tamaulipas registró un flujo de IED de 779 millones de dólares, es decir 144 millones por encima de la cifra del mismo periodo en 2016 (Secretaría de Desarrollo Económico, 2017). Es importante señalar que en el año 2013, se alcanzó una cifra histórica record de 1706 millones de dólares que reflejó claramente los resultados de toda una labor de promoción y vinculación con el exterior de Tamaulipas y que ha seguido hasta ahora.

Si se analiza el desarrollo económico de la zona por el número de empresas en el Programa para la Industria Manufacturera, Maquiladora y de Servicios de Exportación (IMMEX) instaladas en Reynosa, se demuestra que dicha ciudad se ha mantenido por encima de otras ciudades de la región fronteriza. Al cierre del 2015, Reynosa contó con 147 establecimientos, ${ }^{8}$ mientras que las ciudades vecinas de Matamoros y Nuevo Laredo apenas sumaron 142 establecimientos (Tabla 1).

Tabla 1: Número de industrias establecidas en el ámbito nacional, estatal y local

\begin{tabular}{|c|c|c|c|c|c|c|}
\hline \multicolumn{2}{c}{ Número de establecimientos de la IMMEX manufacturera } & \\
\hline Periodo & $\begin{array}{c}\text { Total } \\
\text { nacional }\end{array}$ & $\begin{array}{c}\text { Tamaulipas } \\
\text { total }\end{array}$ & Matamoros & $\begin{array}{c}\text { Nuevo } \\
\text { Laredo }\end{array}$ & Reynosa & $\begin{array}{c}\text { Otros } \\
\text { municipios }\end{array}$ \\
\hline $2007 / 12$ & 5140 & 404 & 133 & 37 & 147 & 87 \\
\hline $2008 / 12$ & 5254 & 397 & 134 & 37 & 143 & 83 \\
\hline $2009 / 12$ & 5214 & 387 & 130 & 35 & 140 & 82 \\
\hline $2010 / 12$ & 5108 & 372 & 122 & 36 & 141 & 73 \\
\hline $2011 / 12$ & 5079 & 361 & 117 & 37 & 138 & 69 \\
\hline $2012 / 12$ & 5104 & 355 & 105 & 33 & 149 & 68 \\
\hline $2013 / 12$ & 5142 & 360 & 110 & 32 & 150 & 68 \\
\hline $2014 / 12$ & 5020 & 355 & 113 & 30 & 148 & 64 \\
\hline $2015 / 12$ & 5006 & 349 & 111 & 31 & 147 & 60 \\
\hline
\end{tabular}

Fuente: Elaboración propia con datos de la SEDET (2016) e Inegi (2016a).

\footnotetext{
${ }^{8}$ Según datos del Inegi (2001) en la publicación: Estadística de la Industria Maquiladora de Exportación 1995-2000, Reynosa contaba con 76 plantas maquiladoras en 1995, mientras que el personal ocupado en ese mismo año era de 39304.
} 
Como resultado de la actividad industrial, Tamaulipas generó a diciembre 2015, un total de 202232 empleos en las maquiladoras de los 2358532 generados a nivel nacional. Solo en Reynosa, se generaron 97118 mientras que en Matamoros 54177 y en Nuevo Laredo 30119 (Tabla 2).

Tabla 2: Empleos generados por la maquiladora a nivel nacional, estatal y municipal

\begin{tabular}{|c|c|c|c|c|c|c|}
\hline \multicolumn{7}{|c|}{ Personal ocupado en la IMMIeX } \\
\hline Periodo & Total nacional & $\begin{array}{l}\text { Tamaulipas } \\
\text { total }\end{array}$ & Matamoros & $\begin{array}{r}\text { Nuevo } \\
\text { Laredo }\end{array}$ & Reynosa & $\begin{array}{c}\text { Otros } \\
\text { municipios }\end{array}$ \\
\hline $2007 / 12$ & 1902121 & 182659 & 51623 & 17901 & 95371 & 17764 \\
\hline $2008 / 12$ & 1738182 & 166455 & 44956 & 17315 & 87277 & 16907 \\
\hline $2009 / 12$ & 1645291 & 144196 & 40803 & 17252 & 70903 & 15238 \\
\hline $2010 / 12$ & 1810482 & 162298 & 46320 & 20350 & 80762 & 14866 \\
\hline $2011 / 12$ & 1879114 & 158205 & 42929 & 18832 & 81037 & 15407 \\
\hline $2012 / 12$ & 1991760 & 170823 & 42093 & 25772 & 86127 & 16831 \\
\hline $2013 / 12$ & 2116022 & 181803 & 46778 & 23046 & 91415 & 20564 \\
\hline $2014 / 12$ & 2242865 & 193551 & 52140 & 26604 & 94489 & 20318 \\
\hline $2015 / 12$ & 2358532 & 202232 & 54177 & 30119 & 97118 & 20818 \\
\hline
\end{tabular}

Fuente: Elaboración propia con datos de la SEDET (2016) e Inegi (2016a).

En febrero de 2016 el número de trabajadores registrados en el Instituto Mexicano del Seguro Social (Imss) en Tamaulipas fue de 605 288. Destacan por su participación, la industria de la transformación con $40.9 \%$ y el comercio con 18.2\%. De diciembre de 2010 a febrero de 2016 el número de trabajadores aumentó a 66 348, lo que representó un incremento acumulado de 12\%. La industria de la transformación registró un crecimiento acumulado de 23\%. Más de la mitad de los trabajadores registrados en el imss se concentró en las regiones de Reynosa y Tampico (Tabla 3). En poco más de cinco años las ciudades que más han crecido son: Reynosa y Matamoros con 16772 y 14186 empleos generados respectivamente (Sistema de información económica de Tamaulipas [sIETAm], 2016). Es importante destacar que al cierre del 2016, Reynosa se mantenía como líder con el mayor número de empleos generados por la industria maquiladora, en diciembre de ese año se cerró con una cifra de 109567 trabajadores, es decir, 12449 nuevos empleos en solo un año (López, 2017).

Otro indicador de impacto en el desarrollo transfronterizo Reynosa-McAllen, lo constituye el hecho de que la zona es considerada la salida al corredor económico más importante del continente en todas direcciones y con todas las oportunidades que ello represente. Un mayor número de industrias en Reynosa significa un incremento del comercio exterior y de cruces internacionales tanto de mercancías como de personas. 
Tabla 3: Empleos registrados en el Imss

\begin{tabular}{|c|c|c|c|c|c|}
\hline \multicolumn{6}{|c|}{ Personal registrado en el IMss } \\
\hline Región & $31 / \mathrm{dic} / 2010$ & $29 / \mathrm{feb} / 2016$ & $\%$ del Total & $\begin{array}{l}\text { Variación } \\
\text { absoluta }\end{array}$ & Variación relativa \\
\hline Matamoros & 99214 & 113400 & 18.7 & 14186 & 14.3 \\
\hline Nuevo Laredo & 70611 & 82740 & 13.7 & 12129 & 17.2 \\
\hline Reynosa & 178261 & 195033 & 32.2 & 16772 & 9.4 \\
\hline Victoria & 54480 & 63838 & 10.5 & 9358 & 17.2 \\
\hline Tampico & 114333 & 127985 & 21.1 & 13652 & 11.9 \\
\hline El Mante & 22041 & 22292 & 3.7 & 251 & 1.1 \\
\hline Total & 538940 & 605288 & 100.0 & 66348 & 12.3 \\
\hline
\end{tabular}

Fuente: Elaboración propia con datos de la SEDET (2016).

En este apartado, las exportaciones e importaciones por las aduanas de Tamaulipas y en específico de Reynosa, manifestaron del año 2010 al 2015 un incremento en sus movimientos anuales con una ligera caída en 2015. Sin embargo, para el primer trimestre del 2016 Tamaulipas ocupó el primer lugar como exportador de manufacturas IMMEx con un monto de 19000 millones de dólares, muy por encima de Chihuahua y Baja California (Flores, 2016). Este incremento de la actividad de entrada y salida de mercancías, produce una serie de servicios logísticos como transporte, servicios aduanales, empaque, embalaje, almacenaje, inventarios que a la vez genera un impacto positivo en ingresos para ambos lados de la frontera por dichos servicios.

\section{Conclusiones}

La paradiplomacia que se ha construido en la región transfronteriza Reynosa-McAllen es producto de las necesidades de ajuste a políticas nacionales e internacionales en materia económica, pero en especial, se origina en la comunicación eficiente entre actores sociales de ambos lados. Se identifica plenamente con una propuesta de desarrollo económico basada en el intercambio y la cooperación eficiente y eficaz entre actores y sectores sociales. Se ha creado, sin duda una simbiosis, término proveniente de la biología que nos habla de una asociación íntima para beneficiarse mutuamente en su desarrollo vital. La SEDET y las dependencias municipales de Reynosa y el organismo MEDC en McAllen, han podido consolidar un proyecto de trabajo conjunto que cuenta con la venia de los gobiernos de ambos países como modelo de desarrollo económico transfronterizo.

A reserva de lo que se pudiera encontrar en una evaluación exhaustiva del TLCAN y del impacto de la globalización económica para la región transfronteriza ReynosaMcAllen, el crecimiento de la actividad económica ha generado una transformación en 
la estructura, infraestructura y equipamiento de estas ciudades, se ha incrementado el empleo y se han mejorado algunos indicadores de bienestar que la convierten en una región competitiva y hasta cierto punto atractiva para los inversionistas en el mercado mundial. En gran medida esta transformación se debe a la actividad paradiplomática, por lo que es necesario seguir con la apertura que han mostrado diversos actores e incrementar la cooperación transfronteriza entre ambos lados.

La historia, cercanía geográfica e interés sobre temas que impactan en ambas naciones, obliga a reforzar el diálogo y la suscripción de acuerdos mutuos. Con la aparición del TLCAN en los años noventa, la dinámica de cooperación y negociación se hizo más latente a través de dependencias gubernamentales y nuevos actores públicos y privados, que a la postre, ha sido la forma de desarrollo económico más congruente para hacer prosperar un territorio abandonado por el gobierno mexicano hasta los años sesenta cuando se crea el Programa Industrial Fronterizo.

La llegada a la presidencia de Estados Unidos de Donald Trump aparentemente marca una nueva época para las relaciones transfronterizas, en especial, cuando el mandatario mira con recelo el TLCAN y se dispone a modificarlo en un afán por encontrar mayores ventajas para los estadounidenses. Uno de los puntos de interés es el de poner un arancel alto cuando las importaciones pasen de un cierto volumen y si el precio interno cae por debajo de un cierto nivel, lo que se ha llamado como snap back (Morales, 2017). Independientemente de lo que resulte de estas negociaciones, los actores entrevistados sostienen que ni la inseguridad, ni el proteccionismo de uno u otro lado bloquearán sus intenciones de cooperar entre ambas fronteras. Lo cierto es que se ha encontrado una especie de fórmula entre actores públicos y privados locales para evitar ser vulnerables a los vaivenes políticos y económicos de cualquier lado y se esmeran para que la relación de cooperación fluya siempre hacia las altas esferas del bienestar transfronterizo.

Esta actitud es positiva hoy en día, sobre todo si se conoce que las dinámicas económicas internacionales son inciertas. La crisis hipotecaria que explotó en el 2007 en los Estados Unidos debido, entre otras cosas, a la complejidad de los mercados financieros, impactó el consumo de muchos de los productos generados en la región transfronteriza Reynosa-McAllen y, a pesar de ello, la economía se ha sostenido. Esto comprueba que la paradiplomacia ha dado frutos y se debe por tanto, seguir apostando a este tipo de dinámicas transfronterizas.

En efecto, la paradiplomacia se encarga de promover y defender la región desde la perspectiva local en aquellos temas aún no resueltos desde la federación que incluye por cierto el grave problema de inseguridad, especialmente en Reynosa y las crisis económicas. El hermanamiento de ciudades es un ejemplo de alta actividad paradiplomática. Gracias a este acercamiento, se logra una sincronía o complicidad entre ciudades a cada lado de la frontera para tratar asuntos comunes y que requieren soluciones conjuntas. En ese contexto, las cámaras de comercio y otros organismos tanto en México como en Estados Unidos se han convertido en aliados clave para apoyar la gestión transfronteriza; sin embargo, es claro que independientemente de los acuerdos interinstitucionales o los llamados acuerdos de hermanamiento, las actividades de vinculación con el exterior de los municipios se seguirán llevando a cabo a pesar de lo que diga y haga el presidente Trump.

El proceso de apertura a partir del consenso de Washington y su proyecto neoliberal globalizador generó la participación de múltiples actores en la esfera 
internacional que rehúyen precisamente al Estado benefactor o el proteccionismo estatal que fue eficaz en el periodo de guerra y posguerra, pero que en la actualidad ha quedado relegado a una entidad regulatoria y vigilante de los procesos sociales. Es común ver ahora a inversionistas, empresarios, representantes de cámaras industriales y comerciales, miembros de la sociedad civil organizada y académicos de ambos lados involucrarse en asuntos públicos que antes eran exclusivos de los Estados nación. Se puede afirmar, que la confianza y compromiso de instituciones y organismos y la visión global de los funcionarios públicos y empresarios de la región Reynosa-Mcallen son las piezas clave en la construcción paradiplomática y en el desarrollo económico de esta región.

Algunas alternativas para continuar con esta dinámica paradiplomática y generar desarrollo y bienestar para esta región son: consolidación de la infraestructura logística para el comercio exterior; mejorar la disponibilidad de servicios para la industria; promover la llegada de industrias que generen valor agregado a los procesos o maquiladoras de última generación; continuar el trabajo de hermanamiento de ciudades y en especial, reconstruir el tejido social en Reynosa por medio de mejores salarios, equipamiento urbano social, proyectos educativos de gran alcance para fortalecer a las juventudes y contar con una visión global en las autoridades de las administraciones públicas.

Sin duda, la actividad paradiplomática es una estrategia de desarrollo regional transfronterizo basada en la cooperación que busca además de un crecimiento económico, un desarrollo y bienestar de la sociedad impactando positivamente en la calidad de vida de sus habitantes en ambos lados. Corre a favor en este esfuerzo, el impacto generado por el gran número de industrias que se han instalado y se pueden instalar en Reynosa aprovechando su posición geográfica, la infraestructura y equipamientos urbanos construidos, el gran número de empleos directos e indirectos generados, la mano de obra especializada y calificada que ya existe, y el desarrollo colateral de negocios que sirven a la industria o sea la proveeduría que ya existe y se puede potenciar todavía más.

Finalmente se subraya el hecho de una cierta cultura de participación de actores locales y estatales en la esfera internacional lo que ha generado conocimientos, capacidades y habilidades en estos y, a su vez, un apoyo invaluable para los gobiernos federales de ambos lados, lo que se traduce en una focalización de necesidades que fácilmente pueden articular hacia afuera de la región. Se vuelve así una actividad complementaria más no opuesta a la relación con la federación y sus políticas hacia la frontera compartida.

\section{Referencias}

Aldecoa, F. y Keating, M. (1999). Paradiplomacy in Action: The Foreign Relations of Subnational Governments. Gran Bretaña, Reino Unido: Psychology Press.

Aldecoa, F. y Keating, M. (2001). Paradiplomacia: Las relaciones internacionales de las regiones. Madrid, España: Marcial Pons, Ediciones Jurídicas.

Alonso, P. (2015). Ficha técnica estado de Texas. Recuperado de http://ucm.gesmail.icex.es/ icex/201506/MI_TEXAS_FichaTecnica_EstadoTexas_2015.pdf 
Amilhat, A. (2016). Gentes y agentes, condiciones paradiplomáticas de la creación de una frontera móvil. En S. González, N. Cornago y C. Ovando (Eds.), Relaciones transfronterizas y paradiplomacia en América Latina. Aspectos teóricos y estudios de casos (pp. 47-71). Santiago de Chile: RIL. Recuperado de http://www.academia.edu/25231337/ Gentes_y_agentes_condiciones_paradiplom $\% \mathrm{C} 3 \% \mathrm{~A} 1$ ticas_de_la_creaci\%C $3 \% \mathrm{~B} 3 \mathrm{n}$ de_una_frontera_m\%C3\%B3vil

Barajas, M. (2013). Cap. 1 La interdependencia como una perspectiva teórica para entender el desarrollo de la región transfronteriza México-Estados Unidos. En M. R. Barajas y L. F. Aguilar (Coords.), Interdependencia, cooperación y gobernanza en regiones transfronterizas (pp. 33-75). Baja California, México: El Colegio de la Frontera Norte.

Barajas, M. (2015). Fronteras, integración y desarrollo transfronterizo. Recuperado de https://archivos.juridicas.unam.mx/www/bjv/libros/9/4320/4.pdf

Comisión Nacional de Inversiones Extranjeras. (2016). Informe estadístico sobre el comportamiento de la inversión extranjera directa en México (enero-diciembre 2016). Recuperado de https://www.gob.mx/cms/uploads/attachment/file/191839/Informe_Congreso-2016-4T.pdf

Consejo Binacional para el Fomento Económico de Reynosa (Cobifer). (2013). Localización estratégica. Recuperado de http://www.cobifer.com/localizacion_estrategica.html

Córdova, G. (2013). Reporte de propuestas generales de acuerdo a sesiones oficiales. Chihuahua, México: El Colegio de la Frontera Norte.

Cornago, N. (2010). La descentralización como elemento de innovación diplomática: causas estructurales y lógicas de acción. En L. Maira (Ed.) La política internacional subnacional en América Latina (pp. 107-134). Buenos Aires: El Zorzal. Recuperado de http://www.academia.edu/2951867/La_descentralizaci\%C3\%B3n_como_elemento_ de_innovaci\%C3\%B3n_diplom $\% \mathrm{C} 3 \% \overline{\mathrm{A}} 1$ tica

Dávila, C., Schiavon, J. y Velázquez, R. (2008). La paradiplomacia de las entidades federativas en México (Documento de trabajo). Recuperado de https://cide.repositorioinstitucional.mx/jspui/handle/1011/468

Duchacek, I. (1986). The Territorial Dimensions of Politics: Within, Among, and Across Nations. Londres, Inglaterra: Westview Press.

Flores, L. (2016). Tamaulipas lidera exportaciones manufactureras. El Economista. Recuperado de https://www.eleconomista.com.mx/estados/Tamaulipas-lidera-exportaciones-manufactureras-20160612-0072.html

García, C. (1996). La actividad exterior de las entidades políticas subestatales. Revista de Estudios Políticos, (91), 235-264. Recuperado de http://www.paradiplomacia. org/upload/downloads/0ad48a174cbc38cafafd11fc8a47db3blaactividadexteriordelasentidadespol\%C3\%ADticassubestatales.pdf

Garza, H. y Chacón, S. (2002). Entre la globalización y la dependencia. La política exterior de México 1994-2000. México: El Colegio de México, Centro de Estudios Internacionales, Tecnológico de Monterrey.

Gobierno del Estado de Tamaulipas. (s.f.). Regiones de Tamaulipas. Recuperado de http://archivo.tamaulipas.gob.mx/gobierno/dependencias/\#

González, G. (2002). Las estrategias de política exterior de México en la era de la globalización. En H. Garza y S. Chacón (Coords.), Entre la globalización y la dependencia (pp. 27-80). México: El Colegio de México, Centro de Estudios Internacionales, Tecnológico de Monterrey. 
Gutiérrez, A. (2013). Local Efforts and Global Impacts: A City-Diplomacy Initiative on Decentralisation. Perspectives: Review of Central European Affairs, 21(2), 49-61. Recuperado de https:/ /www.ceeol.com/search/article-detail?id=244758

Instituto Nacional de Estadística, Geografía e Informática (Inegi). (2001). Estadística de la industria maquiladora de exportación 1995-2000. Recuperado de http://internet. contenidos.inegi.org.mx/contenidos/productos/prod_serv/contenidos/espanol/bvinegi/productos/historicos/2104/702825176211/702825176211_1.pdf

Instituto Nacional de Estadística, Geografía e Informática (Inegi). (2015). Cuéntame, información por entidad del conteo intercensal 2015. Recuperado de http://cuentame.inegi.org. $\mathrm{mx} /$ monografias/informacion/Tam/Poblacion/default.aspx?te$\mathrm{ma}=\mathrm{ME} \& \mathrm{e}=28$

Instituto Nacional de Estadística, Geografía e Informática (Inegi). (2016a). IMMEX y personal ocupado en diciembre 2015. Recuperado de http://www.inegi.org.mx/ sistemas/bie/

Instituto Nacional de Estadística, Geografía e Informática (Inegi). (2016b). Población por entidad federativa y municipios 2010. Recuperado de http://www3.inegi.org. $\mathrm{mx} /$ sistemas/iter/default.aspx?ev $=5$

Kincaid, J. (1999). The International Competence of u.s. States and Their Local Governments. Regional and Federal Studies, 9(1), 111-133. Recuperado de http:// www.tandfonline.com/doi/abs/10.1080/13597569908421074

Lecours, A. (2002). Paradiplomacy: Reflections on the Foreign Policy and International Relations of Regions. International Negotiation, 7(1), 91-114. doi: $10.1163 / 157180602401262456$

Ley sobre Celebración de Tratados. 2 de enero de 1992. Recuperado de http://www. diputados.gob.mx/LeyesBiblio/pdf/216.pdf

López, A. (5 de marzo de 2017). Crece industria Immex en más de 12 mil empleos en el 2016 en Reynosa. Hoy Tamaulipas. Recuperado de http://www.hoytamaulipas.net/notas/285280/Crece-industria-IMMEX-en-mas-de-12-mil-empleos-enel-2016-en-Reynosa.html

Martínez, M. (2011). La diplomacia municipal en México. Cinco estudios de caso. México: Universidad Nacional Autónoma de México.

McAllen Chamber of Commerce. (2016). McAllen Economic Scan. Recuperado de http://mcallen.org/wp-content/uploads/2014/10/market_profile.pdf

McAllen Economic Development Corporation. (s.f.). McAllen-Reynosa Maps. Recuperado de http:/ /www.mcallenedc.org/living-here/mcallen-reynosa-maps\#industrial

Morales, R. (2017). Trump amaga con dejar el TLCAN. El Economista. Recuperado de http://eleconomista.com.mx/industrias/2017/04/19/trump-amaga-dejar-tlcan

Morales, V. y Reyes, C. (2016). Identidades compartidas: la centralidad de los lazos culturales como motor paradiplomático. Desafios, 28(1), 79-120. Recuperado de https://revistas.urosario.edu.co/index.php/desafios/article/view/4472/3208

Oddone, N. (2014). Cooperación transfronteriza en América Latina: Una aproximación teórica al escenario centroamericano desde la experiencia del Proyecto Puertas Abiertas. Revista Oikos, 13(2), 129-144. Recuperado de http://www.revistaoikos.org/seer/index.php/oikos/article/view/375/213 
Oliveras, X. (2014). La acción transfronteriza de los gobiernos locales en un contexto de endurecimiento fronterizo y crisis económica. El caso de la región Tamaulipas-Texas. Carta Económica Regional, 26(113), 13-35. Recuperado de http://www. revistascientificas.udg.mx/index.php/CER/article/view/5482

Oliveras, X., Durà, A. y Perkmann, M. (2010). Las regiones fronterizas: balance de la regionalización de la cooperación transfronteriza en Europa (1958-2007). Documents d'Anàlisi Geogràfica, 56(1), 21-40. Recuperado de http://ddd.uab.cat/ pub/dag/02121573v56n1/02121573v56n1p21.pdf

Plan Estatal de Desarrollo Tamaulipas. (2011-2016). Actualización octubre 2013. (2013). Recuperado de http://transparencia.tamaulipas.gob.mx/wp-content/uploads/2013/11/ III-PED-TAMAULIPAS-Actualizaci\%C3\%B3n-2013-2016.pdf

Primer Informe de Gobierno 2014 José Elías Leal. Presidente Reynosa Tamaulipas. Recuperado de http://www.reynosa.gob.mx/transparencia/informe-anual/INFORME-DE-GOBIERNO-JEL-2014.pdf

Ramos, J. (2002). Gobiernos locales y la cooperación transfronteriza México-Estados Unidos. Espiral, Estudios sobre Estado y Sociedad, IX(25), 107-139. Recuperado de http:// www.redalyc.org/html/138/13802504/

Rhi-Sausi, J. y Oddone, N. (2013). Integración regional y cooperación transfronteriza en los nuevos escenarios de América Latina. Investigación y Desarrollo, 21(1), 260-285. Recuperado de http://www.redalyc.org/html/268/26828624011/

Rodríguez, S. (2006). La paradiplomacia: las relaciones internacionales del gobierno de Chiapas. En S. Rodríguez (Coord.), La paradiplomacia: las relaciones internacionales de los gobiernos locales (pp. 115-147). México: Porrúa.

Sandoval, E. (2007). El espacio económico Monterrey-San Antonio: Coyuntura histórica e integración regional. Frontera Norte, 20(39), 69-99. Recuperado de http://www.scielo. org.mx/scielo.php?script=sci_arttext\&pid=S0187-73722008000100003

Schiavon, J. A. (2006). La proyección internacional de las entidades federativas: México ante el mundo. Cuadernos de Política Internacional, (13).

Secretaría de Desarrollo Económico. (2017). Dos países, una región. Recuperado de http:// www.tamaulipas.gob.mx/desarrolloeconomico/por-que-invertir-en-tamaulipas/

Secretaría de Desarrollo Económico y Turismo (SEDET). (2016). Indicadores estadísticos IMMEX. Recuperado de http://sietam.tamaulipas.gob.mx/bajasietamelecciones-eliminar/ immex.htm

Sistema de Información Económica de Tamaulipas (sIETAM). (2016). Personal ocupado en la IMMEX México y Tamaulipas. Recuperado de http://sietam.tamaulipas.gob.mx/

Texas A\&M Agrilife. (s.f.). Recuperado de http://agrilifecdn.tamu.edu/stxborderinsects/files/2012/05/map.png

Velázquez, R. (2007). La paradiplomacia mexicana: Las relaciones internacionales de las entidades federativas. Publicaciones CIDE, (57), 1-36. Recuperado de http://repositorio-digital.cide.edu/handle/11651/1079

Zeraoui, Z. (2011). Diplomacia paralela y las relaciones internacionales de las regiones. $D a$ safios, 23(1), 59-96. Recuperado de http://www.redalyc.org/pdf/3596/359633169003. pdf 


\section{Entrevistas}

García, R. (23 de mayo de 2016). Comunicación personal. McAllen, Texas.

Ortiz, Á. (30 de mayo de 2016). Comunicación personal. Ciudad Victoria, Tamaulipas.

Karla María Nava Aguirre

Mexicana. Doctora en Ciencias Administrativas por la Universidad Autónoma de Tamaulipas, México. Especialista en Estudios de la Frontera México-Estados Unidos por El Colegio de la Frontera Norte (El Colef), 2016. Profesora e investigadora adscrita al Departamento de Economía de la División de Negocios de la Universidad de Monterrey. Sus líneas de investigación son: vinculación internacional de gobiernos locales; internacionalización de empresas multilatinas; comercio, logística y negocios internacionales. Publicaciones recientes: Nava, K. y Martínez, M. (2016). La internacionalización de los municipios fronterizos de Tamaulipas ¿el pivote geográfico de la acción exterior hacia los Estados Unidos?, 223-262.

Gustavo Córdova Bojórquez

Mexicano. Doctor en Ciencias Sociales por la Universidad Autónoma Metropolitana Unidad Xochimilco. Maestría en Administración Integral del Ambiente por El Colef. Egresado del Centro de Estudios Superiores del Estado de Sonora de la Carrera de Ecología. Profesor e investigador en El Colegio de la Frontera Norte adscrito al Departamento de Estudios Urbanos y del Medio Ambiente, sede Ciudad Juárez, Chihuahua desde abril de 1997 a la fecha. Líneas de investigación: desarrollo humano transfronterizo, manejo de recursos naturales, participación ciudadana. Publicaciones recientes: Córdova, G. y Romo, L. (2015). Espacio urbano y actores sociales en la ciudad de Chihuahua ¿mutua reconfiguración?, El Colegio de la Frontera Norte. 